\title{
Ultrasound of the paediatric chest
}

\section{Goya Enriquez}

(C) Springer-Verlag 2011

US is increasingly used to study the lung parenchyma, pleural cavity, mediastinum and chest wall, both prenatally and postnatally. Foetal lungs are routinely inspected in the standard cardiac four-chamber view. Owing to their fluid content, the foetal lungs are seen as homogeneous structures of medium echogenicity; the echogenicity increases slightly throughout gestation. Using colour or power Doppler, the examiner can assess lung vascularisation without intravenous contrast, a major advantage of US in clinical practice.

Routine use of prenatal US has led to higher detection rates for congenital lung malformations (bronchopulmonary foregut malformations). These are readily identified on US since they are more echogenic than the surrounding lung and produce a mass effect on neighbouring structures, particularly the heart. The malformations tend to be focal and unilateral; hence, a bilateral increase in lung size and echogenicity would rather suggest laryngeal or tracheal atresia. The observation of significant lung enlargement due to accumulated bronchial secretions in foetuses with tracheal atresia inspired prenatal treatment of severe pulmonary hypoplasia secondary to diaphragmatic hernia. This treatment stimulates lung development by artificial tracheal obstruction achieved via endoscopic insertion of an intratracheal balloon. The degree of pulmonary hypoplasia can be determined by calculating the lung-to-heart ratio

Disclaimer Dr. Enriquez has no financial interests, investigational or off-label uses to disclose.

G. Enriquez $(\bowtie)$

Department of Pediatric Radiology,

Vall d'Hebron Children's Hospital,

P. Vall d'Hebron 119-129,

Barcelona 08035, Spain

e-mail: genriquez@vhebron.net
(LHR) using US, or by calculating the relative foetal lung volume (RFLV) using magnetic resonance planimetry. It is extremely difficult to identify the ipsilateral lung remnant ("ipsilateral cap") by US, and therefore it is excluded from the LHR calculation. This limitation of US can sometimes be overcome using power Doppler to follow the pulmonary artery and thereby identify the lung cap.

Unlike the foetal lung, the postnatal lung appears as echogenic lines with a comet-tail configuration produced by air in the alveoli. The most common condition studied by US in clinical practice is lung consolidation. One characteristic US sign of lung consolidation is the sonobronchogram produced by air-filled bronchi within consolidated lung (Fig. 1). The consolidated lung is vascularised by pulmonary vessels, easily identified by their quadriphasic waveform on pulsed Doppler.

US can be used to study several pleural conditions, particularly fluid collections. Classically, when pleural fluid was suspected on plain film, lateral decubitus films were taken to confirm the finding. In our opinion, US should be used instead of decubitus films for this purpose since the fluid can be seen directly, fluid volume calculated and the nature of the fluid characterised to determine the severity of the condition. Thereby, further radiation exposure is avoided.

US is the best radiological technique for characterising the nature of pleural collections, which are classified according to their sonographic appearance into two types: simple and complex. Simple effusions are completely anechoic and usually correspond to transudates. Complex effusions or exudates present one or more of these US features: echogenic debris, fibrin strands, septa and a honeycomb appearance. In children, infections are the most common cause of complex effusions (parapneumonic effusions, empyema). Characterisation of pleural collections 
Fig. 1 Pneumonic consolidation in a child. Chest radiograph (a) shows opacification of the right hemithorax. Transverse sonographic view of the right hemithorax (b) demonstrates that the opacification is due to lung consolidation. Multiple sonobronchograms are visualised
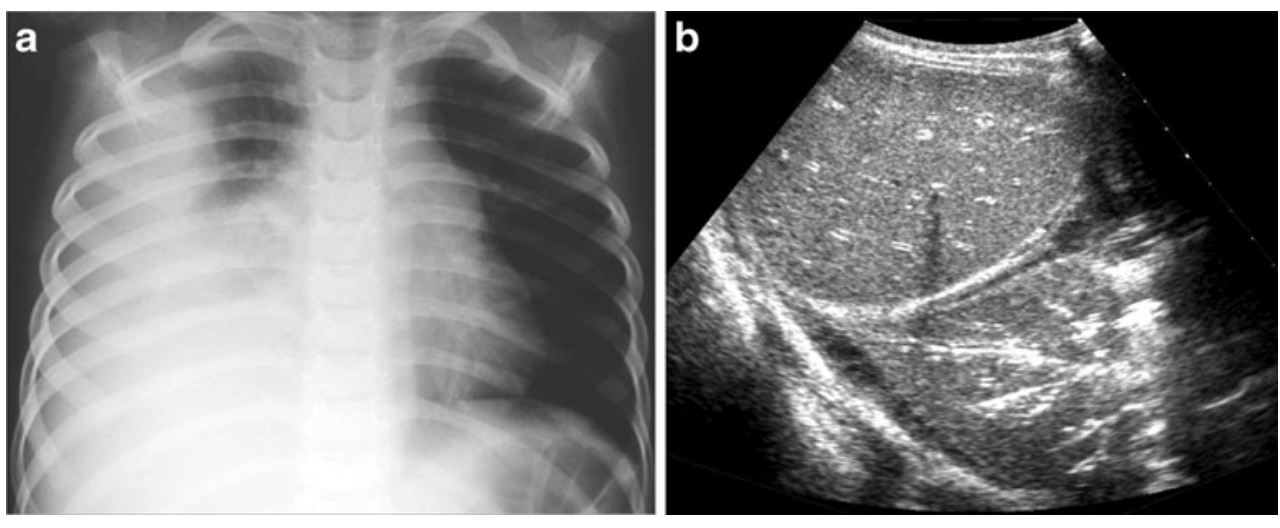

The radiographically opaque hemithorax is another main indication for US, which should be performed before any medical procedure is considered.

In conclusion, sonography is very useful for studying chest abnormalities in children; however, it should not be used for investigating every abnormal plain film finding. We recommend US particularly for lobar pneumonias, suspected pleural effusions, opaque hemithorax, equivocal plain film findings and abnormalities refractory to treatment. 